\title{
Demarcación ética y jurídica entre la asistencia personal y la asistencia sexual en un contexto de vida independiente
}

\section{Manuel García Ortiz}

Facultad de Derecho, Universidad de Castilla-La Mancha

manuel.garcia37@alu.uclm.es

Laguntzaile pertsonalaren irudia oraindik ez dago arauz erabat garatuta, eta sexu-laguntzaren jardueraren euskarri edo lan-esparru izateko arriskutsu gerta daiteke, jarduera hori indarrean dagoen lege-esparruaren mugen baitan dagoelako. Laguntza pertsonala, bizi-proiektu independente baten testuinguruan, ezgaitasunen bat duen pertsonaren autonomia pertsonalerako tresna izateko garatu behar da, eta bere funtzioetan argi eta garbi mugatu, jarduera biak ez daitezen nahas.

\section{GAKO-HITZAK:}

Ezgaitasuna, laguntza pertsonala, bioetika, giza eskubideak, sexu-laguntza.
La figura del asistente personal aún no está plenamente desarrollada normativamente y existe el riesgo de que sirva de cobertura o marco laboral a la actividad de asistencia sexual, al estar esta última en los límites del marco legal vigente. La asistencia personal, en el contexto de un proyecto de vida independiente, debe desarrollarse para servir de instrumento a la autonomía personal de la persona con discapacidad y claramente demarcada en sus funciones para no confundir ambas actividades.

\section{Palabras Clave:}

Discapacidad, asistencia personal, bioética, derechos humanos, asistencia sexual. 
La autonomía es, pues, el fundamento de la dignidad de la naturaleza humana y de toda naturaleza racional.

I. Kant, Lógica

\section{Autonomía personal y vida independiente}

Se suelen utilizar popularmente términos como autonomía, independencia, autodeterminación o vida independiente indistintamente para referirse al deseo de que las personas con discapacidad puedan ejercer sus derechos fundamentales relacionados con la capacidad de tomar decisiones sobre su propia vida, residir donde deseen de forma autónoma y contar con las condiciones adecuadas del entorno y los apoyos necesarios para ello.

Sin embargo, es importante precisar las relaciones entre estas diferentes nociones a la hora de abordar el papel de las personas que prestan un apoyo a las personas con discapacidad en calidad de asistentes, para no crear confusión con otras figuras profesionales ligadas tradicionalmente a la de la persona cuidadora. También parece apropiado realizar una demarcación estricta entre las figuras de asistente personal y asistente sexual.

Tradicionalmente se ha entendido desde un paradigma de la caridad o desde un modelo de intervención médico-psiquiátrico que la persona con discapacidad era "objeto" de cuidados y atenciones por parte de alguien de su entorno familiar o por una persona profesional, y que el papel de ella era ser una receptora pasiva de esos cuidados y tratamientos (Pérez y Chhabra, 2019) y no un ser "sujeto" de derechos.

El enfoque social de la discapacidad en las últimas décadas ha ido configurando un modelo basado en la prestación de apoyos para facilitar el ejercicio de los derechos y libertades, en el que esta figura tradicional y caritativa de prestación de cuidados resulta insuficiente. Desde este enfoque de derechos humanos no se parte de una concepción unidimensional de la persona con discapacidad reducida a sus características biológicas, sino que se parte del supuesto de que la discapacidad es uno más de los aspectos, pero no el único, que forman parte de su realidad y que, ante todo, la persona está dotada de la misma dignidad inherente que tiene cualquier ser humano y el ejercicio de sus derechos fundamentales es el bien superior al que debe atender cualquier acción, por encima de consideraciones científicas o médicas incluso. La discapacidad es una situación que se produce cuando hay un desajuste entre el entorno y las características individuales de la persona que conduce a una relación de desventaja en ese entorno. Es esta situación de desventaja la que genera situaciones de vulneración de sus derechos y es la que la sociedad tiene la obligación de corregir.

En lo que se refiere a la autodeterminación, la Real Academia de la Lengua Española la define como la capacidad de una persona para decidir por sí misma algo. Es decir, que la autodeterminación implica la capacidad de discernimiento en el ámbito del análisis de la realidad y el ejercicio de la libertad en la toma de alternativas responsables sin la tutela de otra persona. Por supuesto, se trata de una cuestión muy subjetiva y relativa, ya que dicha capacidad puede tener diferentes grados de desarrollo en cada persona, en distintos contextos de desenvolvimiento social o en diferentes momentos de la vida, al igual que se pueden dar diferentes facilitadores y barreras que generen dificultades especiales para el ejercicio de la autodeterminación. Desde este punto de vista, pocas personas con o sin discapacidad podrían reclamar una capacidad de autodeterminación completa y de manera absoluta en todo momento de su vida.

La autodeterminación implica ejercicio consciente de la libertad individual, una compleja cuestión filosófica. A lo largo de la historia del pensamiento, el problema del ejercicio de la libertad ha sido uno de los temas centrales de la filosofía y nos ha proporcionado una enorme cantidad de literatura y argumentos desde los que abordar la cuestión de la autonomía y la autodeterminación. De hecho, existen tantos puntos de vista contrapuestos que resulta casi imposible elegir un determinado planteamiento sin que surjan aporías y contradicciones planteadas por otros pensadores. En cualquier caso, podemos partir de cierto consenso en torno a que el ejercicio de la autonomía en la toma de decisiones requiere de las condiciones de posibilidad mínimas para poder ejercerla. Damos por supuesto que una persona privada de iniciativa, proactividad, o conocimiento del medio en que vive, o que no tiene la capacidad para plantearse objetivos a alcanzar por cualquier motivo, no está en condiciones de ejercer la autonomía personal por no tener capacidad de autodeterminación.

Sin embargo, aunque la autodeterminación debe entenderse, por tanto, como una condición a priori de la autonomía, no debería establecerse como tal de una forma absoluta y permanente, ya que el proceso que lleva a ella como capacidad del ser humano se puede favorecer muchas veces a través de factores ambientales que permiten a la persona construir esa capacidad de libertad cognitiva y desarrollar en gran medida el control sobre sus deseos y voluntad; en definitiva, la capacidad de dirigir libremente su intención hacia los fines que se propone. De hecho, en el proceso de la construcción social de un colectivo como vulnerable, como es el caso de las personas con discapacidad, suele haber muchos elementos que se interrelacionan y retroalimentan. Una persona aislada y privada de autonomía, difícilmente cuenta con las condiciones de posibilidad para desarrollar la capacidad de autodeterminación en igualdad de condiciones con el resto de la sociedad. La reclusión, la indefensión, la segregación, el abandono o la sobreprotección son barreras para el desarrollo de la autodeterminación y la capacidad para la autonomía personal. Favorecer 
entornos inclusivos, libres de barreras y con apoyo adecuado es necesario en personas con y sin discapacidad, y no solamente para las personas que ya tienen capacidad de autogobierno, sino especialmente también para aquellas que aún no han desarrollado esa capacidad totalmente.

Desde el modelo de atención centrado en la persona (Vilà i Mancebo, 2019) se propone la contraposición de los conceptos de autonomía al de heteronomía y el de independencia al de dependencia, entendiendo que una persona puede ser dependiente en la medida en que precisa de apoyos para la realización de actividades de su vida cotidiana, pero autónoma a la hora de tomar decisiones sobre cuestiones vitales con plena capacidad de comprensión del significado y las consecuencias de sus decisiones. Se entiende, por tanto, que la circunstancia de ser dependiente, en caso de contar con apoyos adecuados, no es un obstáculo al ejercicio de la autonomía personal. La vida independiente es el resultado práctico del ejercicio de esa autonomía a partir del despliegue de un proyecto vital en el marco de una sociedad.

En gran medida, la figura de la persona cuidadora tradicional, desde un modelo de atención médico o caritativo, ha sido una prestadora de servicios que ha tratado de resolver las necesidades más básicas de la persona casi a un nivel de mera supervivencia, pero que no ha generado autonomía, y los motivos de ello tienen que ver seguramente con concebir a las personas con discapacidad, no como seres humanos completos en todos sus aspectos, sino solamente como un conjunto de "síntomas", "deficiencias", "carencias", etc. Como objetos y no como sujetos.

Por tanto, entendemos la vida independiente como la consecuencia deseable del ejercicio de la autonomía personal cuando se dan los factores personales (la autodeterminación) y ambientales necesarios para ello. Solo en un contexto de vida independiente la persona puede ejercer completamente sus derechos, desarrollar su proyecto vital y construir plenamente su identidad.

\section{La figura de la persona asistente personal}

Tras un largo proceso, que comienza décadas antes, de reconocimiento gradual de los derechos de las personas con discapacidad, en el año 2006 la Asamblea General de la ONU aprueba la Convención Internacional sobre los Derechos de las Personas con Discapacidad. La Convención, ratificada por 182 países, hace explícitos los derechos y establece la obligación por parte de los estados firmantes de promover, proteger y garantizar el pleno disfrute de todos ellos por parte de este grupo social.

Con carácter general, la Convención está desarrollada desde el enfoque de derechos del modelo social de la discapacidad. Ello se traduce en que es prioritaria la intervención sobre el entorno de la persona, principalmente creando espacios, productos y servicios desde una filosofía de diseño universal, con el fin de no presentar obstáculos a un amplio rango de diversidad humana. También actuando sobre los ya existentes para acometer actuaciones de accesibilidad de ajuste y prestando el apoyo necesario mediante el uso de tecnologías y la asistencia personal.

El artículo 19 de la Convención consagra el derecho de las personas con discapacidad a la vida independiente y a ser incluidas en la comunidad, a poder elegir su lugar de residencia y dónde y con quién vivir en igualdad de condiciones con las demás personas, y para ello que los estados firmantes faciliten que tengan acceso a una variedad de servicios de apoyo a la autonomía personal, incluida la asistencia personal que sea necesaria para facilitar su existencia y su inclusión en la comunidad y para evitar su aislamiento o separación de esta.

Posteriormente, el Comité de los derechos de las personas con discapacidad, como órgano de la ONU de expertos independientes que supervisa la aplicación de la convención, ha realizado una serie de recomendaciones a los estados firmantes sobre su aplicación y desarrollo. En su observación general número 5 (2017), Sobre el derecho a vivir de forma independiente y a ser incluido en la comunidad, referida al citado artículo 19 de la convención, el Comité desarrolla la figura del asistente personal de forma más explícita estableciendo que "La asistencia personal se refiere al apoyo humano dirigido por el interesado o el ‘usuario’ que se pone a disposición de una persona con discapacidad como un instrumento para permitir la vida independiente", establece una relación personal y una financiación personalizada en base a las necesidades de apoyo que se deben proporcionar, controlado de forma directa por la persona con discapacidad. Los asistentes personales deben ser contratados, capacitados y supervisados por las personas que reciban la asistencia, y no deben ser "compartidos" sin el consentimiento pleno y libre de cada una de estas personas. La Comisión aborda en la recomendación directamente la cuestión de que "el concepto de asistencia personal en que la persona con discapacidad no ejerce plenamente la libre determinación y el control de sí misma no se considerará conforme con el artículo 19”.

Es decir, la asistencia personal es un servicio claramente diferenciado de la prestación dirigida a la atención domiciliaria de personas dependientes y de la figura tradicional de la persona "cuidadora", con la que suele haber una relación mediata y cuya prestación de servicios no depende directamente de la persona con discapacidad habitualmente. En cualquier caso, aunque el asistente personal preste "cuidados", en caso de que sea necesario, sus funciones no se limitan necesariamente a estas cuestiones ni al ámbito puramente doméstico.

En España, la figura de la persona asistente personal está recogida expresamente en la Ley 
39/2006, de 14 de diciembre, de Promoción de la Autonomía Personal y Atención a las personas en situación de dependencia de España. La conocida significativamente como "Ley de dependencia" y no como "Ley de autonomía personal” y que, efectivamente, ha tenido un desarrollo desigual en relación con los servicios domésticos de apoyo a las personas dependientes y de asistencia en las actividades más básicas de la vida diaria, frente al desarrollo de la figura del asistente personal como facilitadora del ejercicio de la autonomía personal.

Aún no existe un desarrollo claro de la figura de asistencia personal, ya sea a través de un certificado de profesionalidad o de una titulación concreta, y por parte del movimiento representativo del grupo social hay una demanda constante de la completa definición y puesta en marcha de los servicios de asistencia personal y la dotación presupuestaria suficiente para su adecuada prestación.

\section{Problemas éticos y legales planteados en la figura de asistencia sexual}

Al contrario que la figura de asistencia personal, recogida en la legislación española y en los tratados internacionales, la de asistencia sexual no está configurada legalmente y, de hecho, se mueve en los límites de la normatividad. El principal problema que se plantea en torno a la figura de la asistencia sexual surge por la confluencia con la figura tradicional de la persona prostituida. Existe un debate sobre el tema y en la Unión Europea hay diferentes posiciones legales que van de la prohibición a la regulación (Heim y Monfort, 2015). En la prostitución el cuerpo humano es considerado una mercancía cuya poseedora, la persona que se prostituye o es prostituida, pone a disposición de la clientela a cambio de una remuneración. Esta explotación está tradicionalmente ligada a una relación de proxenetismo con una tercera persona 0 a relaciones de indefensión frente a la clientela por parte de personas en situación de vulnerabilidad, por lo que se dan habitualmente situaciones de menoscabo de la dignidad humana y es considerada frecuentemente una actividad perniciosa para la sociedad. Desde posicionamientos feministas de defensa de los derechos humanos, se proponen diferentes medidas encaminadas a la protección de las personas que ejercen la prostitución o a la abolición de la práctica. En contra de este punto de vista se suele defender la libertad para decidir de la persona que se prostituye como única propietaria de su cuerpo (Garaizábal, 2008).

Algunos de los primeros derechos subjetivos desarrollados en la historia tienen que ver con la propiedad privada del propio cuerpo, tanto en los procesos de emancipación de la esclavitud, como en la noción de "tener tu cuerpo" (habeas corpus), que aparece en la Common law inglesa. Hacia el final de la Edad Media existían aún diferentes sistemas judiciales superpuestos y no era raro que se extrajese una confesión como reo acusado o sospechoso de un delito mediante la tortura por parte de un tribunal determinado. De hecho, el papa Inocencio IV en 1252 autorizaba el uso de la tortura para obtener la confesión durante los procesos inquisitoriales mediante la bula papal Ad extirpanda (Parra López, 2013). En ella recomendaba no llegar hasta provocar la muerte del reo o su mutilación en el transcurso del proceso de obtener la confesión. Hacia 1568, el juez Dyer, presidente de la Corte de Súplicas Comunes de Inglaterra, liberó un prisionero acogido al derecho de habeas corpus por haber sido forzado a prestar juramento. En la fundamentación de la sentencia, el juez justificó su decisión alegando que "nemu tenetur seipsum prodere", nadie puede ser obligado a producir evidencia contra sí mismo. En esta sentencia tendrá su antecedente remoto la célebre quinta enmienda ${ }^{1}$ a la Constitución de los Estados Unidos de 1791 (Holdsworth, 1912). El derecho a la posesión del cuerpo se inicia como la protección de los cuerpos de la ciudadanía frente a los excesos de los poderes tradicionales y del naciente Estado, y se consagra en los siguientes siglos, culminando en los derechos derivados de esta noción. Con frecuencia el liberalismo que protagonizará la lucha por las libertades en esta primera generación de reivindicación de los derechos humanos fundamentará sus principios sobre la base de la propiedad privada del propio cuerpo, un derecho que procede del "estado de naturaleza" y del que se derivarían todos los demás. La abolición de la esclavitud, como hito de las revoluciones liberales, fortalece esta idea de la propiedad sobre el propio cuerpo. Ninguna persona puede ser comprada ni vendida. Toda persona es soberana y propietaria de sí misma.

El derecho a la propiedad del propio cuerpo está consagrado en nuestros sistemas constitucionales y en los tratados internacionales de derechos y libertades desde hace al menos dos siglos, y este derecho puede ser legítimamente esgrimido en contra del ejercicio de la prostitución y, por tanto, la persona puede ejercer ese derecho "a no ser vendida". Sin embargo, de este derecho se suele entender en ocasiones (como un corolario a la propiedad privada del propio cuerpo) que también se puede renunciar a él, y que no entra dentro del abanico de "derechos irrenunciables". Que, en definitiva, la persona que se prostituye hace con su cuerpo lo que ella desea en calidad de propietaria (Beltrán Pedreira, 2015).

Un derecho irrenunciable es aquel que no puede dejar de ser ejercido, ya que su abandono implica lesionar indirectamente el mismo derecho en una persona en una situación más vulnerable. De esta manera, muchos derechos de segunda

1 "Ninguna persona estará obligada a responder de un delito castigado con la pena capital, o con cualquier otra pena, salvo en la presencia o acusación de un Gran Jurado [...]; ni se le forzará a declarar contra sí misma en ningún juicio criminal; ni se le privará de la vida, la libertad o la propiedad sin el debido proceso legal; ni se ocupará su propiedad privada para uso público sin una justa indemnización”. 
generación relacionados con el trabajo se consideran irrenunciables con el fin de que no se establezcan las condiciones para la explotación de otras personas al renunciar, por ejemplo, a los periodos de descanso 0 al salario mínimo establecido.

En relación con la propiedad privada del propio cuerpo, los tratados internacionales y la legislación de la mayoría de los países tienden a considerar la propiedad del cuerpo como un derecho irrenunciable. No está permitida la esclavitud voluntaria, ni la venta de órganos ni, en la mayoría de los países, la maternidad subrogada. Actualmente, en España existe el delito de prostitución forzada, pero tanto el ejercicio de ella como su consumo no están explícitamente penados. Sin embargo, el proxenetismo está castigado con penas de prisión de dos a cinco años y multa de doce a veinticuatro meses (Ley Orgánica 1/2015) y las Administraciones desarrollan políticas activas en contra de su ejercicio, con independencia de la eficacia de tales medidas, ya que se entiende que es ejercida de forma mayoritaria por mujeres bajo coacción y en condiciones de explotación.

Por otro lado, la Ley Orgánica 2/2010 en el artículo 3 nos dice que "todas las personas tienen derecho a adoptar libremente decisiones que afectan a su vida sexual y reproductiva sin más límites que los derivados del respeto a los derechos de las demás personas y al orden público garantizado por la Constitución y las Leyes", lo que deja el espacio abierto tanto a la prostitución como a la asistencia sexual en la medida en que estas actividades se realicen de manera voluntaria, sin lesionar los derechos de otras personas y sin alterar el orden público.

En lo que se refiere a la asistencia sexual, en principio pretende diferenciarse de la prostitución y ser ejercida sin coacción y bajo condiciones de dignidad para la persona asistente, prestando un abanico de servicios que no incluirían solamente ni necesariamente la actividad sexual. La asistencia sexual está habitualmente justificada en el derecho a la sexualidad de las personas con discapacidad, entendiendo como parte de este derecho el de tener placer sexual.

No existe actualmente un consenso científico sobre si las relaciones sexuales son una necesidad biológica, aunque sí sobre los beneficios que proporcionan habitualmente tanto en el orden físico como en el psicológico, ni un posicionamiento jurídico claro sobre si forma parte de los derechos sexuales y reproductivos (De Asís, 2017), entre los cuales no aparece recogido en los tratados internacionales y que tienen más que ver con el derecho a la no discriminación y el acceso a los recursos públicos y tecnológicos. En cualquier caso, tiende a considerarse por parte de la sociedad que todo aquello no está expresamente prohibido es en sí mismo un derecho universal, pero nuestros sistemas legales positivos, como la Constitución Española, reservan la categoría de derecho fundamental a un abanico de ellos entre los que no están algunos que podrían considerar como tales, como la justicia o sanidad gratuita o la educación no obligatoria. La noción de derecho es considerada, con carácter general, de forma más extensa por parte de la ciudadanía profana que por parte de las personas expertas y profesionales del derecho.

Observando el desarrollo de la figura en los países de nuestro entorno (Martínez Ochoa, 2016), encontramos que Suiza, Dinamarca, Bélgica, Holanda, Luxemburgo y Alemania tienen regulaciones en materia de asistencia sexual de forma expresa o implícita. En Europa (Míguez, 2019), solamente Suiza, en el cantón de Ginebra ha regulado legalmente la creación de empresas de asistencia sexual, exigiendo a los asistentes una diplomatura universitaria de la rama sanitaria. Su actividad es profesional y remunerada, y además se intenta separar emocionalmente a la persona asistente y asistida para no establecer vínculos permanentes, al estilo de la deontología sanitaria. Suiza, por su parte, tiene legalizada la actividad de la prostitución, por lo que se establecen de facto dos figuras claramente separadas entre la persona que se prostituye y aquella que presta asistencia sexual.

En Alemania, donde la prostitución está legalizada desde 2002, lo que en principio abre la puerta a una asistencia sexual entendida como especialización de ella, existe el Instituto para la autodeterminación de las personas con discapacidad (ISSB), que ofrece dos tipos de servicio: asesoramiento sexual y acompañamiento sexual. En el primer caso se trata de proporcionar información sobre sexualidad a la persona, mientras que el segundo servicio es "preparatorio" para las prácticas sexuales, en un marco de desarrollo del erotismo facilitador de la actividad sexual, pero diferenciado de la actividad de asistencia.

En Bélgica, Holanda, Dinamarca y algunos territorios de Australia, la asistencia sexual para personas con discapacidad está subvencionada por el Estado para algunas personas con discapacidad, pero en ocasiones en un marco de alegalidad de la persona que ejerce este papel y de forma indistinguible del uso de la prostitución. En el caso de Holanda y Dinamarca, se da la circunstancia de que la prostitución está legalizada y regulada, por lo que esta proporciona el marco legal del ejercicio de la asistencia sexual como ocurre en Alemania; en Bélgica en 2018 se reguló la figura del asistente sexual de forma diferenciada, ya que la prostitución es ilegal en este país.

En Francia, donde la prostitución es ilegal, tras un agitado debate parlamentario sobre la figura del asistente sexual en 2012 se concluyó por parte de la comisión parlamentaria al efecto que no se podía admitir que la ayuda sexual sea un derecho creado que implique una obligación por parte de la sociedad, debiendo quedarse en el marco de 
las iniciativas individuales y voluntarias (Comité Consultatif National d'Éthique, 2012). Sin embargo, en febrero de 2020 el presidente Macron ha afirmado públicamente que se va a estudiar legalizar la figura de la asistencia sexual (Efe, 2020). En otros países, como Reino Unido, la prostitución es ilegal, por lo que la asistencia sexual se mueve en el marco de la desregulación y en el límite de la clandestinidad.

Por tanto, encontramos tres escenarios posibles: países abolicionistas como España, donde el ejercicio de la prostitución no es ilegal pero sí lo es el proxenetismo y se buscan políticas activas para su desaparición, países donde la prostitución está expresamente prohibida y países en los que la prostitución es legal y está regularizada. Por otro lado, y partiendo de este marco, legislaciones en las que la asistencia sexual puede considerarse una profesión diferenciada de la prostitución, otros en las que se emplea la regulación de la prostitución como cobertura legal a modo de una especialización profesional, o países en los que se considera una actividad voluntaria y no profesional.

\section{Límites en el ejercicio de la asistencia personal}

La relación de asistencia personal es flexible entre la persona asistente y asistida, por lo que las funciones exactas a desempeñar se concretan en un contrato bilateral entre las partes, para atender las necesidades y preferencias de cada persona concreta. Como en toda relación laboral, cualquier cláusula ilegal en un contrato de trabajo es nula de facto, pero en el caso de incluir tareas de asistencia sexual nos encontraríamos en un vacío legal al no estar desarrollada normativamente la figura. En la medida en que la venta y consumo de sexo no es ilegal en España, no se trataría, en principio, un ilícito, pero el desarrollo normativo de la figura del asistente personal puede acabar dando cobertura legal a la de asistente sexual a falta de ese desarrollo. También puede darse la circunstancia de que pueda considerarse que se incurre en un delito de trata con fines de explotación sexual si se entiende que, empleando violencia, intimidación o engaño, o abusando de una situación de superioridad o de necesidad o vulnerabilidad de la víctima se ha obligado a la persona a realizar actos sexuales (Código Penal, artículo 187). Explícitamente, el Código Penal considera que existe explotación sexual, incluso con consentimiento de la víctima, cuando la persona se encuentra "en una situación de vulnerabilidad personal o económica” (Ley Orgánica 5/2010, art. 48). En este caso se considera
A la hora de establecer los límites en el desempeño profesional del asistente personal y el deslinde con las funciones de asistencia sexual, la figura legal de la objeción de conciencia puede ser una de las que aparezcan en el debate en torno a las posibilidades que se pueden abrir.
Hay razones que hacen pensar que la objeción no es el mecanismo más adecuado para regular las competencias profesionales de aquella persona que no desea en ningún caso realizar funciones de asistente sexual estando contratada en calidad de asistente personal. La objeción de conciencia es ya de por sí un "derecho" peculiar: el derecho a no cumplir con una obligación (Montoya-Vacadíez, 2014). Una figura muy excepcional, ya que consiste en la posibilidad de no acatar una obligación de carácter público o realizar un acto al que se está obligado por el ejercicio de la profesión elegida invocando motivos éticos o religiosos que entren en conflicto con esa obligación y, estrictamente hablando, solamente puede ejercerse frente a un deber ineludible. De hecho, las primeras regulaciones que aparecen en el mundo se refieren a la obligación de realizar el servicio militar o a ser llamado a filas por el ejército. Incluso en el ejercicio de la profesión médica hay voces discordantes que consideran que el ejercicio de la profesión no es un deber ineludible y, por tanto, no debería poder invocarse la objeción de conciencia para evitar realizar procedimientos contrarios a los propios principios morales. En realidad, la persona que invoca la objeción de conciencia no pretende invalidar la norma, sino que busca la excepción para sí misma por un motivo de conciencia que le genera una contradicción imposible de resolver. Una situación extraña cuando las funciones de la relación laboral están personalizadas a las necesidades de cada persona. En cualquier caso, el derecho a la objeción sanitaria está recogida en diferentes tratados internacionales y en las regulaciones de los colegios profesionales. Sin embargo, en dos actividades que no están aún plenamente desarrolladas y reguladas, parece que sería una vía más sencilla simplemente desligar las funciones de asistencia sexual de las del asistente personal para evitar ese tipo de conflictos éticos que se pueden presentar con frecuencia.

En el caso del o la asistente personal, las funciones habituales tienen que ver con el apoyo a las actividades básica de la vida, como la higiene, el vestido, la deambulación, etc., de las actividades instrumentales como las tareas propias del hogar como la limpieza, cocina, etc., acompañamientos y desplazamientos fuera del domicilio, como puede ser ir al médico, a realizar trámites administrativos, al puesto de trabajo o de estudio, etc., y actividades de esparcimiento como acompañamiento a visitar a familiares, amistades o participar en actividades de ocio de cualquier tipo. Al margen de estas funciones ordinarias, en circunstancias extraordinarias pueden tener la función de prestar primeros auxilios mientras llega asistencia sanitaria durante una emergencia médica o colaborar en algunos procedimientos sanitarios simples y que la propia persona o su entorno inmediato se practicaría a sí misma, así como para la que la persona asistente puede prestar apoyo sin sustituir el papel de un profesional de la salud.

Puesto que se trata de la prestación de un servicio integral y la sexualidad forma parte inseparable y no 
diferenciada de la vida de una persona, atendiendo además a la demanda por parte del grupo social de las personas con discapacidad de no ser considerados seres asexuados, la asistencia personal debe dar en ocasiones apoyo a actividades que están relacionadas directamente con el sexo, como puede ser el vestido, la higiene, el desplazamiento a una cita, la transferencia de un lugar a otro, facilitar elementos físicos para la práctica sexual, etc.; en definitiva, participar en la preparación de la actividad sexual de la persona asistida en el marco que ella elija desde su libertad sexual y en el marco del acuerdo firmado entre ambas personas. En ningún caso se confunde este tipo de apoyo con ser una parte protagonista del acto, “objeto" de la relación sexual ya sea masturbando o llevando a cabo otras prácticas sexuales con la persona asistida.

En el momento en que la asistencia personal se objetiviza sexualmente podría estar renunciando a la soberanía sobre su propio cuerpo, perdiendo su dignidad al ser utilizada o incluso sentirse víctima de acoso sexual por parte de la parte contratante. En el peor de los casos, puesto que el consentimiento se considera irrelevante si media una situación de necesidad o vulnerabilidad, se podría incurrir en un delito de trata con fines de explotación sexual.

\section{Conclusiones}

En el capítulo IV de la Fenomenología del espíritu de Hegel, titulado "Autonomía y dependencia de la autoconciencia: dominio y servidumbre", el filósofo alemán desarrolla uno de los tópicos más importantes para el pensamiento posterior, la así llamada "dialéctica del amo y el esclavo". En el texto, Hegel despliega un diálogo en el que subyace la idea de que la autoconsciencia de un individuo se desarrolla siempre en el marco de un mutuo reconocimiento. Para Hegel no es posible que una autoconciencia se reconozca sin que sea reconocida por otra autoconciencia. Para este ejercicio es necesario, a su vez, reconocer como tal individuo autoconsciente a la otra parte, en un ejercicio de espejos reflejados el uno en el otro que dota de entidad e identidad a ambos sujetos. Se considera que este texto ha tenido una gran influencia en el desarrollo de la noción de lucha de clases en el movimiento obrero, pero también en el desarrollo del concepto de derechos humanos universales en el sentido del mutuo reconocimiento como sujetos de derechos entre distintas personas (DRI, 2010).

La implementación de la figura del asistente personal tiene como fin último el reconocimiento de la dignidad humana inherente a las personas con discapacidad. La figura de asistencia es la facilitadora de la autonomía para una vida independiente y para el ejercicio de las libertades y derechos fundamentales por parte de las personas que, sin ese apoyo, ven restringida su participación a la hora de involucrarse en situaciones vitales.
Sin embargo, como en la dialéctica hegeliana, la relación entre persona asistida y asistente se basa en el mutuo reconocimiento de ambas como seres humanos dotados de dignidad. Ambos deben ser sujetos y nunca objetos, ni de atenciones y cuidados, caritativos o médicos, la persona con discapacidad, ni la persona asistente objeto de acoso, abuso o condiciones de trabajo que entren en conflicto con sus principios morales. Se trata de dos sujetos de derechos en una relación contractual de prestación de servicios. Una relación entre iguales.

La dignidad de la persona con discapacidad no puede obviar o construirse desde la objetualización de la persona asistente. Si bien es cierto que a veces se entiende la figura de la persona asistente como "una mera extensión" de la voluntad de la persona asistida, dicha voluntad no debería entrar en conflicto con la dignidad como persona trabajadora de la otra. Durante siglos, la persona con discapacidad ha sido considerada un mero objeto, bien de caridad, bien de intervención médica, y ha sido el propio movimiento de las personas con discapacidad el que ha reclamado la transición de ese modelo a un "modelo social" en el que pasasen a ser sujetos de derechos, derechos que se reclaman como universales y, por tanto, no pueden dejar de atribuirse y ser extensivos también a la asistencia personal en el ejercicio de sus funciones en el mutuo reconocimiento entre ambos sujetos.

Cierto es que actualmente no se plantea por parte del grupo social la inclusión de tareas de asistencia sexual entre las de asistencia personal, pero la posibilidad de que la regulación de la asistencia personal sirva de cobertura legal a la asistencia sexual, o a que se planteen contratos con cláusulas en este sentido, supone una posibilidad que debe abordarse desde la ética y el marco legal vigente, probablemente regulando claramente las obligaciones y límites de actuación del asistente personal en relación con esa esfera de la vida de la persona con discapacidad. En definitiva, dejando claro no solamente qué sea la asistencia personal, sino también qué es lo que no es.

El desempeño de la asistencia personal debe desarrollarse desde el mutuo reconocimiento y construcción de una identidad basada en la dignidad de ambas personas vinculadas por una relación laboral. Aun sin entrar a valorar la validez de la figura de asistencia sexual, debe entenderse como una construcción completamente diferente de la del asistente personal, por lo que deberían estar claramente diferenciadas. La sexualidad es sin duda una parte central del ser humano y su desarrollo un derecho que debe estar previsto como una de las dimensiones de la persona con discapacidad que tiene que poder disfrutar. La discapacidad y la sexualidad no pueden entenderse como esferas separadas e incompatibles de la vida de un individuo, y en torno a la sexualidad hay actividades de la vida diaria en las que el apoyo de la persona asistente personal debe intervenir como facilitadora, 
como pueden ser el desplazamiento, la higiene o el vestido, pero sin que la propia persona asistente sea objetualizada y se produzca una situación que se consideraría un delito de acoso sexual en el desempeño de cualquier otra profesión.

La legalidad de ambas figuras profesionales es claramente diferente. Mientras que la de la asistencia personal está recogida en tratados internacionales y en leyes con rango de ley orgánica en el estado español, la de asistencia sexual no depende de ningún derecho reconocido ni está prevista en la legislación. La Ley Orgánica 2/2010, de 3 de marzo, de salud sexual y reproductiva y de la interrupción voluntaria del embarazo recoge el derecho a la "salud sexual", y en el artículo 5 afirma que los poderes públicos garantizarán "la eliminación de toda forma de discriminación, con especial atención a las personas con algún tipo de discapacidad, a las que se les garantizará su derecho a la salud sexual y reproductiva, estableciendo para ellas los apoyos necesarios en función de su discapacidad". Sin embargo, no debe entenderse el derecho a la salud sexual como el derecho a tener sexo, sino a la no discriminación en materia de salud. De hecho, la figura de la asistencia sexual, en la medida en que puede confluir con la de profesional de la prostitución, puede suponer que su profesionalización, de mediar cualquier particular, empresa o asociación, incurrir en un ilícito de trata de personas con fines de explotación sexual, incluso si la asistencia sexual se hace voluntariamente. Una alternativa propuesta, por ejemplo, por el Comité Consultivo Nacional de Ética de Francia consiste en mantener esta actividad como relación voluntaria entre particulares.

En cualquier caso, es probable que en los próximos años asistamos a un debate público sobre estas cuestiones para las que, como suele ocurrir en casi todas las cuestiones bioéticas, no es sencillo pronunciarse y establecer posicionamientos sencillos y tajantes o soluciones simples para problemas muy complejos y que implican derechos humanos en conflicto. 
AGENCIA EFE (2020): “Francia estudia legalizar la asistencia sexual a personas con discapacidad", La Vanguardia, 11 de febrero, <https://www.lavanguardia.com/ vida/20200211/473464662538/francialegalizar-asistencia-sexual-discapacidad.html〉.

BELTRÁN PEDREIRA, M. E. (2015): “El cuerpo humano. Derechos sin propiedad o propiedad sin derechos", Revista de estudios políticos, $\mathrm{n}$ 169, pp. 137-166.

COMITÉ CONSULTATIF NATIONAL D'ÉTHIQUE (2012): Vie affective et sexuelle des personnes handicapées Question de l'assistance sexuelle, 〈https:// www.ccne-ethique.fr/sites/default/files/ publications/avis_ndeg118.pdf>.

DE ASÍS, R. (2017): “¿Es la asistencia sexual un derecho?”, Revista Española de Discapacidad, 5 (2), pp. 7-18.

DRI, R., (2010): “La dignidad del sujeto”, La dignidad humana. Filosofía, bioética y derechos humanos, Secretaría de Derechos Humanos del Ministerio de Justicia, Seguridad y Derechos Humanos de la Nación, República Argentina, http://www2.congreso.gob.pe/sicr/cendocbib/ con4_uibd.nsf/E4F6A6FF94D478FC05257D1600 74301C/\$FILE/ceboax-0170.pdf.

HEIM, D. y MONFORT, N. (2005): "Vigilar y castigar: las nuevas propuestas de políticas públicas para la prostitución en Europa; análisis de los modelos de Suecia y los Países Bajos", Revista Nueva Doctrina Penal, 2005/B, Buenos Aires, Editores del Puerto, pp. 771-812.

GARAIZÁBAL, C. (2008): “Los derechos de quienes ejercen la prostitución", VII Jornadas de Pensamiento Crítico, diciembre de 2007, Página Abierta, 191, 4-2008.
GOBIERNO DE ESPAÑA (1995): Código Penal, Artículo 187, BOE núm. 281, de 24/11/1995.

- (2010) Ley Orgánica 5/2010, artículo 48.

- (2015) Rúbrica del Capítulo V del Título VIII del Libro II redactada por el número ciento uno del artículo único de la Ley Orgánica 1/2015, de 30 de marzo, por la que se modifica la Ley Orgánica 10/1995, de 23 de noviembre, del Código Penal (BOE, 31 marzo).

HEGEL, G.W.F. (1982): Fenomenología del Espíritu, España, Fondo de Cultura Económica.

HOLDSWORTH, W. (1912): A History of English Law, T. 1, p. 305.

MARTÍNEZ OCHOA, I. (2016): “La figura del asistente sexual dentro del asistente personal: orígenes, marco legal en España e implantación de su figura en la sociedad”, 〈http://www.fderechoydiscapacidad. es/wp-content/uploads/2018/02/TFM-IIMARINEZ-OCHOA.pdf $\rangle$.

MÍGUEZ, M.N. (2019): “Discapacidad y sexualidad en Europa. Hacia la construcción del acompañamiento sexual”, Revista Española de Discapacidad, 7 (I), pp. 133-152.

MONTOYA-VACADÍEZ, D.M. (2014): Mitos y realidades sobre la objeción de conciencia en la praxis médica, Revista Ciencias de la Salud, 12(3), pp. 435-449.

ORGANIZACIÓN DE NACIONES UNIDAS (2017): Observación general núm. 5 sobre el derecho a vivir de forma independiente y a ser incluido en la comunidad, Comité sobre los Derechos de las Personas con Discapacidad.

PARRA LÓPEZ, E; CASADO, M.A. (2013): La Inquisición en España. Agonía y abolición, Madrid, Los Libros de la Catarata. 
PÉREZ, M.E. y CHHABRA, G. (2019): “Modelos teóricos de discapacidad: un seguimiento del desarrollo histórico del concepto de discapacidad en las últimas cinco décadas", Revista Española de Discapacidad, 〈https://www.cedd.net/redis/ index.php/redis/article/download/429/339/s.

SAIZ, V., MANTINI, M. y GÓMEZ-PAN, P. (2015): “Prostitución, moral sexual y explotación”, Revista Contexto, № 13.
VILÀ I MANCEBO, A. (2019): Derechos y deberes de las personas mayores en situación de dependencia y su ejercicio en la vida cotidiana, Estudios de la Fundación Pilares para la Autonomía Personal, № 6, 〈https://www.fundacionpilares. org/publicaciones/fpilares-estudioo6derechos-deberes-personas-mayores.php〉. 